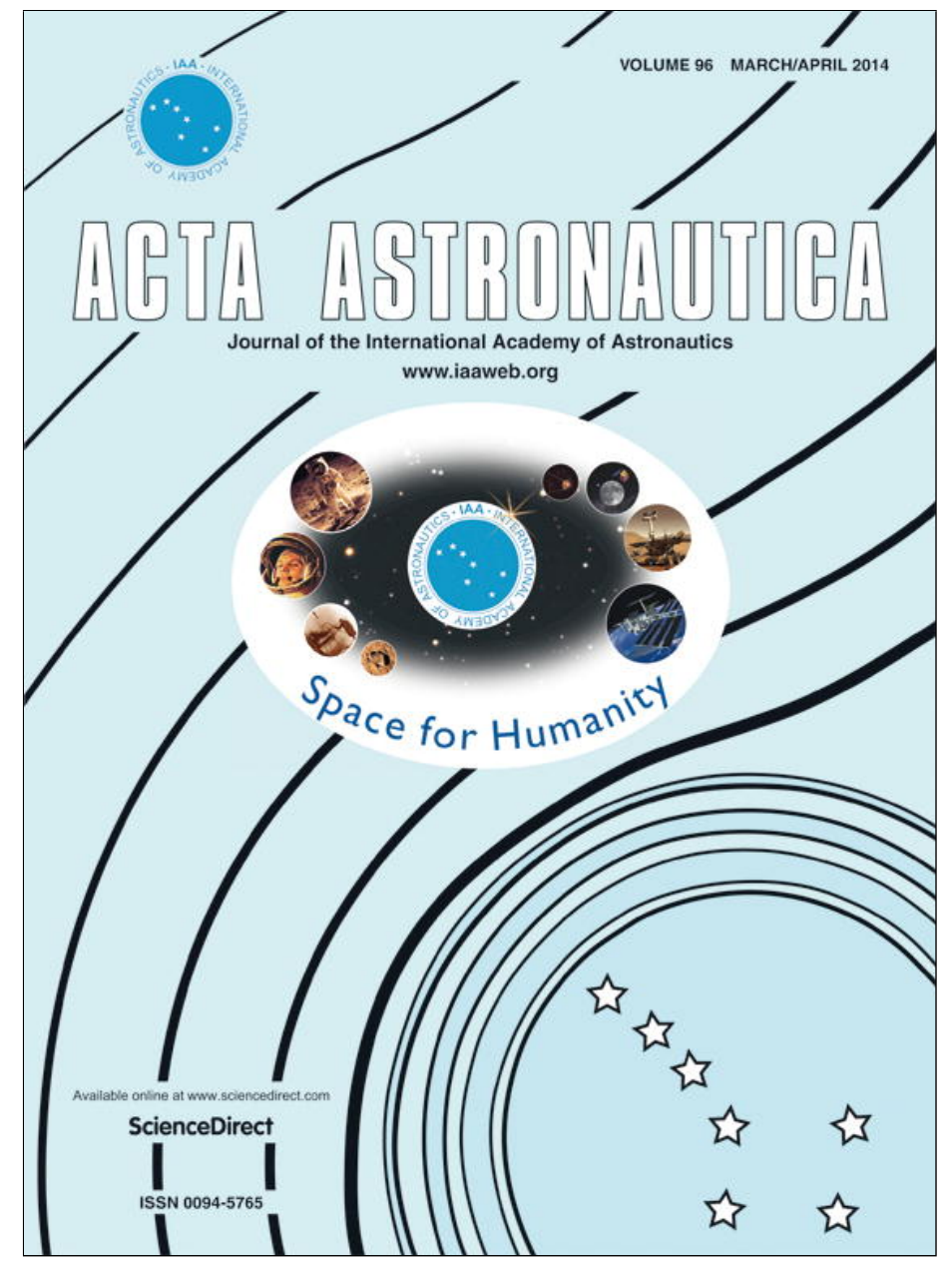

This article appeared in a journal published by Elsevier. The attached copy is furnished to the author for internal non-commercial research and education use, including for instruction at the authors institution and sharing with colleagues.

Other uses, including reproduction and distribution, or selling or licensing copies, or posting to personal, institutional or third party websites are prohibited.

In most cases authors are permitted to post their version of the article (e.g. in Word or Tex form) to their personal website or institutional repository. Authors requiring further information regarding Elsevier's archiving and manuscript policies are encouraged to visit: 


\title{
Greenhouses and their humanizing synergies
}

\author{
Sandra Haeuplik-Meusburger ${ }^{\mathrm{a}, *}$, Carrie Paterson ${ }^{\mathrm{b}}$, Daniel Schubert ${ }^{\mathrm{c}}$, \\ Paul Zabel ${ }^{\mathrm{C}}$ \\ a Vienna University of Technology, Austria \\ b California State University, United States \\ ${ }^{\mathrm{c}}$ Deutsches Zentrum für Luft- und Raumfahrt e.V. (DLR), Germany
}

\section{A R T I C L E I N F O}

\section{Article history:}

Received 5 February 2013

Received in revised form

22 November 2013

Accepted 23 November 2013

Available online 1 December 2013

\section{Keywords:}

Greenhouse design

Habitat integration

Mobile Plant Cultivation Subsystem

Plant-human relationship

Habitability

\begin{abstract}
A B S T R A C T
Greenhouses in space will require advanced technical systems of automatic watering, soilless cultivation, artificial lighting, and computerized observation of plants. Functions discussed for plants in space habitats include physical/health requirements and human psychology, social cohesion, as well as the complex sensorial benefits of plants for humans. The authors consider the role of plants in long-term space missions historically since 1971 (Salyut 1) and propose a set of priorities to be considered within the design requirements for greenhouses and constructed environments given a range of benefits associated with plant-human relationships. They cite recent research into the use of greenhouses in extreme environments to reveal the relative importance of greenhouses for people living in isolated locations. Additionally, they put forward hypotheses about where greenhouses might factor into several strata of human health. In a recent designin-use study of astronauts' experiences in space habitats discussed in Architecture for Astronauts (Springer Press 2011) it was found that besides the basic advantages for life support there are clearly additional "side benefits" for habitability and physical wellbeing, and thus long-term mission success. The authors have composed several key theses regarding the need to promote plant-human relationships in space, including areas where synergy and symbiosis occur. They cite new comprehensive research into the early US Space Program to reveal where programmatic requirements could be added to space architecture to increase the less quantifiable benefits to astronauts of art, recreation, and poetic engagement with their existential condition of estrangement from the planet. Specifically in terms of the technological requirements, the authors propose the integration of a new greenhouse subsystem component into space greenhouses-the Mobile Plant Cultivation Subsystem-a portable, personal greenhouse that can be integrated functionally into future greenhouse constructions in space.
\end{abstract}

(c) 2013 IAA. Published by Elsevier Ltd. All rights reserved.

\section{Introduction}

On Earth, plants grow in different forms according to their natural environment. In extreme environments

\footnotetext{
* Corresponding author.

E-mail addresses: haeuplik@hb2.tuwien.ac.at

(S. Haeuplik-Meusburger), carriep@rof.net (C. Paterson), daniel.schubert@dlr.de (D. Schubert), Paul.Zabel@dlr.de (P. Zabel).
}

technology has to substitute for the Earth's natural conditions in order to allow plants to grow. One of the bestknown and so-far largest endeavors of creating an artificial and closed-ecological biosphere is the Biosphere 2 project in Arizona. In the longest mission, eight people spent 2 years living in the sealed ecosystem. Reported problems include troubles with $\mathrm{CO}_{2}$ and oxygen levels leading to an unbalanced fauna and flora; participants also experienced hunger and interpersonal conflicts [1]. The Biosphere 2 
experiment eventually failed, but results and experiences are still considered relevant for studies on possible future agriculture systems, as well as their importance and effects in isolated environments and beyond Earth [2].

Artificial biospheres and greenhouses will be essential for future human space exploration. As space missions become longer, more arduous, or remote bases for longterm habitation on the Moon or Mars are designed, the effects of micro-gravity, as well as how well different plants grow in space and what kind of light they need are among the many technical issues to be discovered [3]. In addition, near-future missions to the Moon offer a great potential for testing and evaluating those delicate systems.

Besides the technical and scientific challenges, the "human system" becomes equally important. This paper deals mainly with the plant-human relationship in space. It provides a short overview of the history of technical greenhouses in space, highlighting aspects that are related to the humanizing aspects of such systems for sterile, technical environments such as a spaceship, leading to the proposal of an additional greenhouse sub-system component.

The key issues presented confirm the need for personal greenhouse systems. These require technical integration into communal greenhouse projects for the wellbeing of the whole community in future human space habitats.

\section{Greenhouses and gardening in space: a history of systems and benefits}

Salyut 1 (launched April 1971) was the first space station that implemented a greenhouse-it was named Oasis. Since then, plant growth facilities were implemented in the later Salyut stations 4 (1974), Salyut 6 (1976) and Salyut 7 (1982); on Mir (in the module KRISTALL launched in 1990); and on the International Space Station. The US space laboratory Skylab (launched March 1973) only had an educational experiment with space grown rice seeds [4].

Table 1 details the greenhouse facilities that have been used on-board particular space stations.

A number of experiments with seeds and plants have been conducted, with the first plants carried to space in 1960 with Sputnik 4 ([5] p. 3). However the first spacegrown vegetables were reportedly eaten in 1975 onboard Salyut 4. During their mission the cosmonauts Vitali Sevastyanov and Pyotr Klimuk were given permission to eat some of the onion tubers [10].

The first successful life-cycle from seed planted in orbit to flowering plant to producing new seeds was conducted in the growth chamber Phyton on Salyut 7 ([9] p. 177).

\subsection{The first individual greenhouses}

Very early during the Salyut missions, astronauts experimented with plants and "designed" their own little greenhouses. Robert Zimmerman writes that Salyut 6 cosmonaut Valery Ryumin "had a green thumb" and "turned the space station into a veritable jungle by growing [plants] in empty film cassettes, equipment casings, and food containers hung everywhere on the station's walls." ([11] p. 3).

Salyut 6 and 7 cosmonauts even had multiple choices of greenhouses. Valentin Lebedev stayed 211 days onboard the Salyut 7 station and during his long-term stay he planted peas in Oasis, Arabidopsis in the Fiton, lettuce in the Biogravistat, tomatoes and coriander in the Malakhit and onions in a Vazon ([12] p. 169; [13]).

Based on the many experiments with greenhouses, the Soviets recognized very early the psychological benefits of

Table 1

Overview of greenhouse facilities used in space stations. Sources: [5-9]

\footnotetext{
Small Plant Growth Facilities onboard SALYUT $(1,4,6,7)$ and MIR

Oasis 1 (Salyut 1): first plant growth system

Oasis 1M (Salyut 4): improved water metering system

Oasis 1AM (Salyut 6): designed for long duration missions

Oasis 1A (Salyut 7): advanced lighting system

Malachite (Salyut 6): ornamental plant culture system to provide psychological comfort

Fiton (Salyut 6): greenhouse for onions and radish

Svetoblock (Salyut 6): plant system that could be mounted to a light in the cabin

Svetoblock-M (Mir) Svetoblock-S Svetoblock-G Svet (Mir): first joint Russian-US experiment

Magnetogravistat (Salyut 7, Mir): greenhouse for wheat and flax

Biogravistat (Salyut 7): greenhouse for lettuce

Vazon (Salyut 6, 7 and Mir): system for the cultivation of bulbous plants without artificial lighting

Phyton (Salyut 7): miniature growths system, first seed to flower produced on orbit.

Small Plant Growth Facilities onboard STS and ISS

Plant Growth Unit - PGU (STS): plant growth unit that fitted into a mid-deck locker on the Space Shuttle

Plant Growth Facility - PGF (STS): improved lighting and control system

Astroculture System (STS, Mir): closed chamber

Advanced Astroculture System (ISS): student-designed experiment and commercial payload

Plant Generic Bioprocessing Apparatus - PGBA (STS): included fluorescent lighting

Biomass Production System - BPS (STS): developed for long duration missions

LADA (ISS): modular type system

MagISStra, Veggie and AstroGarden: as described below.
} 


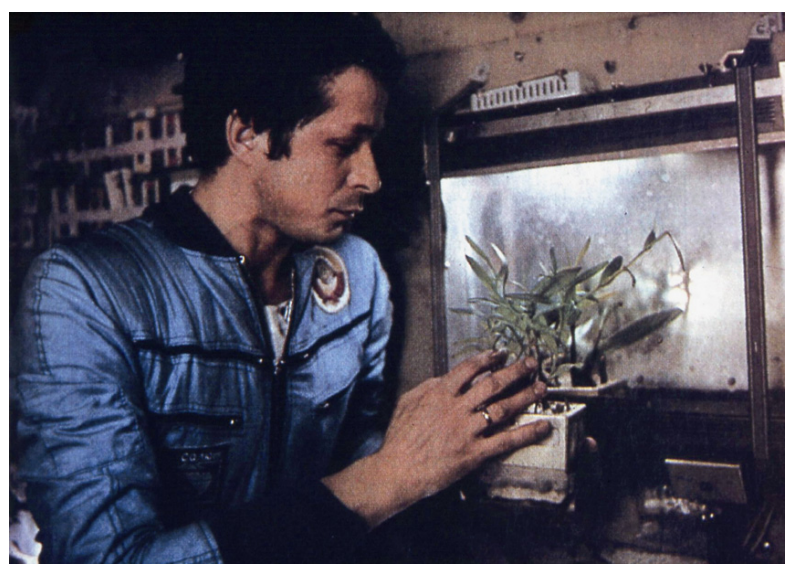

Fig. 1. Expedition 5, cosmonaut Victor Savinykh with plants onboard Salyut 6 (credit: Spacefacts, J. Becker).

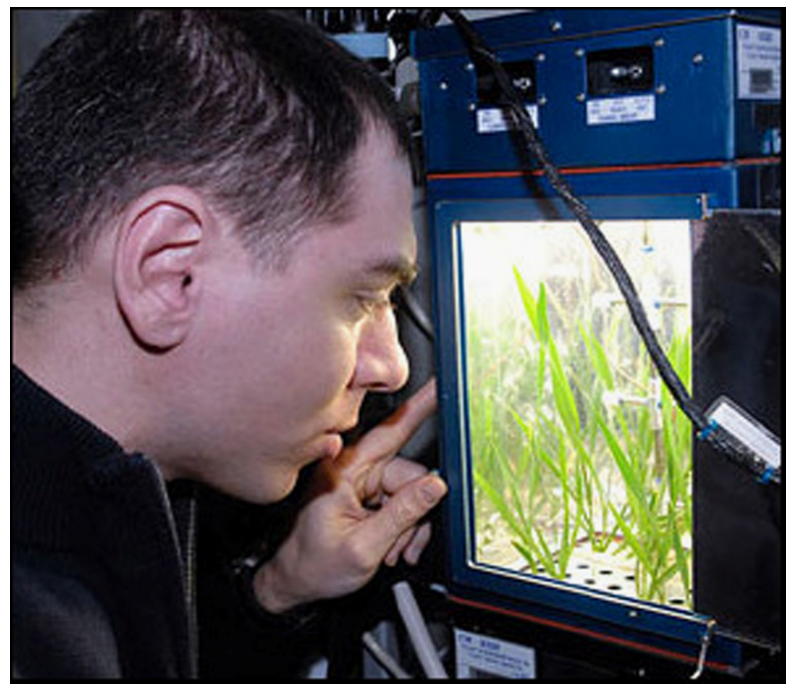

Fig. 2. Russian cosmonaut Sergei Volkov checks the progress of a growth experiment with the LADA-01 greenhouse in the Zvezda Module of the ISS (credit: NASA)

plants (Fig. 1) and designed a device "for the sole purpose of ornamental plant culture to provide psychological comfort to the cosmonauts in the interior of the station."

([5] p. 187).

Since then an international crew of astronauts have used a number of small greenhouses intended for growing vegetables and herbs, for reasons having to do with many aspects of human health.

\subsection{The LADA system and veggie system - food supplements in space}

Today the LADA System (Fig. 2) is used onboard the International Space Station. It is a fully automated, small greenhouse garden and was developed by the Space Dynamics Laboratory at Utah State University and the Institute of Biomedical Problems in Moscow. It has been used on-board the International Space Station (in the Zvezda module) since 2002 . The system is as the size of a standard suitcase and includes a control module $(24.1 \times 17.8 \times$ $24.1 \mathrm{~cm}^{3}$ ). It is sent to the station, already containing root media, or root modules [14].

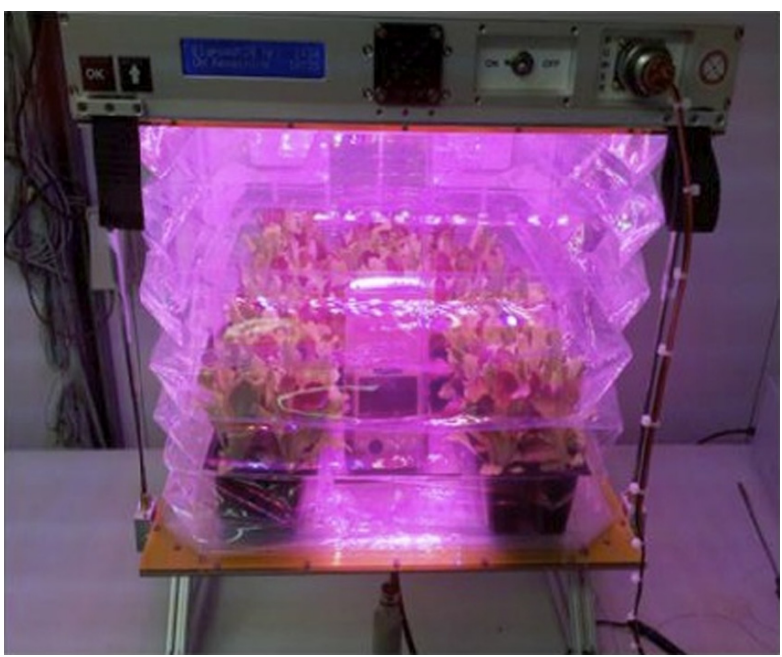

Fig. 3. Veggie System to provide ISS astronauts with fresh salad (credit: Orbitec.com)

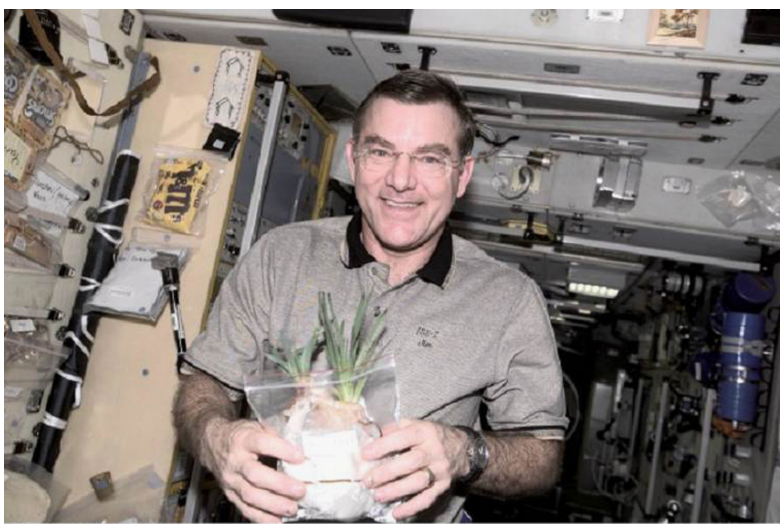

Fig. 4. Jim Voss with Onions on ISS, 2001 (credit: NASA).

One of the LADA System's functions has been to grow edible vegetables for the crew. Gail Bingham from Utah State University calls the LADA System "a salad machine." ([11,5] p. 7).

ORBITEC has been awarded with two NASA contracts to support the development and flight of Veggie (Fig. 3), a deployable growth system to grow salad for long-term mission astronauts [15].

Besides the clear nutritional benefit, astronauts have appreciated the food-growing activity itself. Space Dynamics Lab engineer Shane Topham recounted that following the Columbia disaster, "the crew members who were on the space station were obviously shaken up, and one of the things that the Russian space program did to try and calm them down was to assign them more crew time to gardening because they noticed it did have a calming effect." [16].

\subsection{The Astro Garden - connections to Earth}

Three shuttle flights after the Columbia disaster, on STS-118 in August 2007, a small plant growth chamber was transported to space for an in-orbit experiment. The "Astro 
Garden" was designed as a kind of "hobby kit" where herbs, flowers, and vegetables could be grown. It consists of a growth medium with watering valve and expandable plastic top to keep all parts of the garden enclosed. In parallel, the similarly designed "Space Garden" was made available to schools (Fig. 4).

Astro Gardens are NASA's answer to the need for astronauts to have personal greenhouses. These serve not only as recreation. "During missions, astronauts often long for the tastes and smells of home," says the brochure distributed by the US government's Astro Garden developer. This was also confirmed by several astronauts interviewed by Haeuplik-Meusburger [17]. "Experiments involving plants have provided them with that important link back to Earth, and have made plants an important tool for their psychological well-being in space." [18].

Howard Levine, project scientist for NASA's International Space Station and Spacecraft Processing Directorate, has made comparisons between prison inmates and the life of the astronaut: "It can be pretty harsh out there [in space], confined to a small metal box... Caring for a plant every day provides vital psychological relief, giving astronauts a small remembrance of Earth." [19].

\subsection{The MagISStra development of a "Greenhouse in Space"}

During the STS-120 mission ESA astronaut Paolo Nespoli conducted an experiment with a small educational greenhouse similar in design to the Astro Garden. Nespoli's "Greenhouse in Space" project, proposed and conceived by ESA's Directorate of Human Spaceflight, was a fifteen week scientific experiment conducted in tandem with eight hundred schoolchildren aged between twelve and 14 years old at Cite de l'espace in Toulouse France; ESA European Astronaut Centre, Cologne, Germany; ESA ESRIN, Frascati, Italy; and Ciencia Viva in Lisbon, Portugal. Observations were to be made about the germination and growth of Arabidopsis thaiana, a common flowering plant and model organism that has been studied in space since the first days of Salyut experiments. Nespoli's seeds germinated but quickly developed a potentially hazardous fungus necessitating the seeds be disposed of; meanwhile on Earth, the children and a Mars500 crew completed their part of the mission with much more promising results ([20] p. 3).

One lesson from this milestone experiment suggests the benefits of having complementary experiments performed by diverse groups of people on Earth in coordination with space-bound experiments. Although these do not have a one-to-one correlation, much can be learned in the dialog that connects humans on Earth and humans in space, especially where studying the human-plant relationship is an objective.

\subsection{Spontaneous gardening: the case of STS-61-A}

Similar to the early cosmonauts, NASA and ESA astronauts have appreciated all forms of plants during their travel, even before the first greenhouses arrived. Fig 4 shows Expedition Two flight engineer Jim Voss in the
Zvezda Service Module of the International Space Station ISS holding a plastic bag with two large onions.

Earlier, in 1985, Wubbo Ockels instigated an unplanned greenhouse activity during his mission on STS-61-A. In addition to the planned experiment with corn seeds he had ten extra seeds and made his personal greenhouse with a piece of plastic foam in a plastic bag with a zipper, and a knife for making holes into it. After a few days the leaves grew a few centimeters. He said the crew then had a "little party" and everybody ate a small amount of fresh food. The whole procedure to make the greenhouse took him 10 min. [21].

Ockels' innovation process confirms the importance and relevance of previous gardening activities of cosmonauts. As well, it indicates the immediacy of the growing environment and ease of incorporating plants into astronauts' daily lives, no matter if highly structured as part of experiments, or just for personal interest and health.

\subsection{Gardening for pleasure: the Salyut and Mir Eras}

Early during the Salyut missions it was learned that plants and dealing with the greenhouse had a positive psychological effect on cosmonauts. During the Salyut and Mir Era, to boost morale and fight against loneliness or depression, surprise leisure activities were organized for the crew from the ground, and gifts were sent with the Progress freighters [7]. In 1979, the Salyut 6 cosmonauts Valery Ryumin and Vladimir Lyakhov received flowering tulips and a kalanchoe plant growing in a Vazon as a gift ([12] p. 155). According to Zimmerman, they were so happy they gave the plant a name: "life tree." [11].

Anecdotal references show that besides their regular duties, astronauts also enjoyed doing experiments with plants and observing them grow. One of the Salyut cosmonauts placed his sleeping bag next to an Oasis greenhouse, in order to see the plants progress immediately after waking up. During the Shuttle-Mir missions Mike Foale seemed not only to like the plant experiments, but for him it was also a "very encouraging" activity, as he reported after the seeds he had planted showed sprouts with leaves ([17] p. 213; [22] p. 82).

\section{Towards planetary greenhouses}

Greenhouses designed for extra-terrestrial environments will be fundamentally different from Earth greenhouses due to technical and scientific requirements. A large external greenhouse on the Moon, for example, will have to incorporate micrometeoroid and radiation shielding because of environmental conditions [17,31]. On Mars, atmospheric pressure is 16 times less than that of Earth. Plants have adapted poorly to hypobaric conditions in studies, showing that a decrease in pressure can cause plants' internal regulatory systems to falter; even in an environment of nearly $100 \%$ humidity a plant may experience dehydration because, as water escapes plants faster in low atmospheric pressures, this causes them to close their stomata, and they "react as if they are dying of thirst." ([23] p. 188). On spaceships, species will also need to be selected that will able to survive the 
oxygen-rich environments fit for humans, as gases nonessential to humans like nitrogen would ideally be eliminated from payload requirements for cost and weight ([23] p. $187 ;$ [3]).

All extraterrestrial greenhouses-even if the facilities will have similar functions to those on Earth-will be designed according to the different gravity conditions. Plants have been grown in micro-gravity but still not on a planetary body. The challenges presented by one-sixth Earth gravity on the Moon or one-third Earth gravity on Mars will affect the movement of water, heat and gases differently in each case [3]. A microclimate "could be generated locally around plant bodies, which would affect gas transport," according to one study by JAXA [24]. Using "convective mixing" gases can be distributed in greenhouses according to a number of variables, including the Grashof number (Gr), proportional to the gravity of a planetary surface; the placement and temperature of hot and cold generators within an extraterrestrial greenhouse could thus be calculated with precision [24].

A soil-based system similar to Earth might be used for planetary greenhouses, or hydroponics (which have a light-weight growing medium), or aeroponics, where no medium is used. Aeroponics and hydroponics are both advantageous for spacecraft systems because of their minimal weight.

Many researchers favor a soil-based system because of its capability to use in situ resources on the Moon and Mars and the possibilities of recycling waste products $[25,26,2,27]$. Yamashita, et al. suggested that using even the barren regolith of Mars will enable a "more than $100 \%$ closure of materials recycled in the life support system." [24].

A cost comparison between closed and open loop systems shows another clear advantage in using available soils and recyclable biomaterials in space agriculture. However aiming toward $100 \%$ closure in the loop requires higher control (and, one could assume, investment) "or such a system will be inevitably unstable," [28] which could detrimentally affect human living conditions. Yamashita, et al. have made the argument that in the case of developing "sustainable habitation on Mars" it will take at least 20 years and 100 people to develop the agricultural system in phases and to accumulate system components. The start time for such operations is a question of debate, with estimations ranging between 2030 and 2050 [29].

In the case of Mars, the challenge for soil-based systems is the difficulty in achieving soil-water flow in a gravity of $0.38 g$; studies by Maggi and Pallud have shown that "Martian gravity reduced water and [nitrogen] solute leaching by about $90 \%$ compared to Earth," leading to an increase in $\mathrm{CO}_{2}, \mathrm{~N}_{2} \mathrm{O}$, and $\mathrm{N}_{2}$ emissions but also " $90 \%$ less water consumption .... and "a substantially lower nutrient supply" needed due to increased nitrates [29]. The resulting effect could mean there would be a " $60-100 \%$ greater concentration" of microbial biomass under Mars-like conditions compared to Earth conditions [30]. Nelson reports that "a living soil contain[ing] a complex microbial ecosystem ... helps prevent the buildup of trace gases or compounds and thus assists with air and water purification." [25].
Another issue on extraterrestrial planets and moons is atmospheric pressure. In general, lower pressure makes the construction of greenhouses easier and more economical due to the lesser material requirements. According to Casado, "A greenhouse operating at reduced internal pressure would require thinner walls and would suffer smaller leaks, since it would be bearing a smaller overpressure." ([23], p. 187). Lower-weight transparent materials could also be used to skin the greenhouse, needed for the light and solar energy transfer that increases the bounty of plant growth.

There are several goals for the food system in planetary greenhouses. In the first place, bioregenerative life support systems that include food minimize and eventually eliminate volume and mass from Earth supplies and increase safety for humans and mission success [31,2,32]. Integrated food production is part of the same closed loop system that assists in regeneration of air and water [31].

Another goal in the design of planetary greenhouses is to increase the quality of food for future astronauts on Moon or Mars bases. This requires nutrition, choice, and variation. At the moment there are only select crops considered for long term space missions. "Ten pick-andeat vegetable crops have been identified ... [including] lettuce, spinach, carrots, tomatoes, green onions, radishes, bell peppers, strawberries, fresh herbs and cabbages." $[33,14]$.

Food quality will have a large impact on crew morale and the success of a long-duration mission [31]. According to anecdotal reports, "healthier and tastier foods decrease the stress often experienced by the crew. This suggests that taste, menu variety, and an array of textures, colors and flavors can contribute to the psychosocial wellbeing of the crew." [33].

\section{Plant life cycles in a technical greenhouse}

Many institutions are currently undertaking research into the use of greenhouses in future space missions and extreme Earth environments. The Mars Society uses the Mars Desert Research Station to simulate conditions on Mars, the University of Gelph in Ontario researches longterm crops like soybeans and barley, Dr. Cary Mitchell of Purdue University works on vertical urban farming with a potential use in space, and research on psychological benefits is being conducted at various South Pole stations [34].

In the following section, issues concerning plant life cycles and relevant system components as developed by the German Space Agency (DLR) are detailed.

\subsection{Plant life cycle in a technical greenhouse}

When considering higher plants for cultivation within a closed environment like a planetary greenhouse, several aspects need to be considered. The first issue relates to seeds. In a technical greenhouse at a research station, seeds are usually planted directly into the planting media, not germinated in a separate system. For a future system on Mars, however, the system may need to be more similar 
to a commercial greenhouse on Earth, where seeds are germinated in a separate system, then planted.

Second, in order to achieve maximized edible biomass output within an extra-terrestrial greenhouse module, optimal plant growth is paramount, which results in the need for individually customisable sets of environmental conditions (e.g. light, nutrient mixture, temperature, and $\mathrm{rH}$ ), and these need to be optimally controlled for each step of the plant life-cycle (Fig. 5). For example, lettuce grows optimally at $73 \% \mathrm{rH}$ and at a temperature of around $20^{\circ} \mathrm{C}$. Furthermore, it requires only low light levels

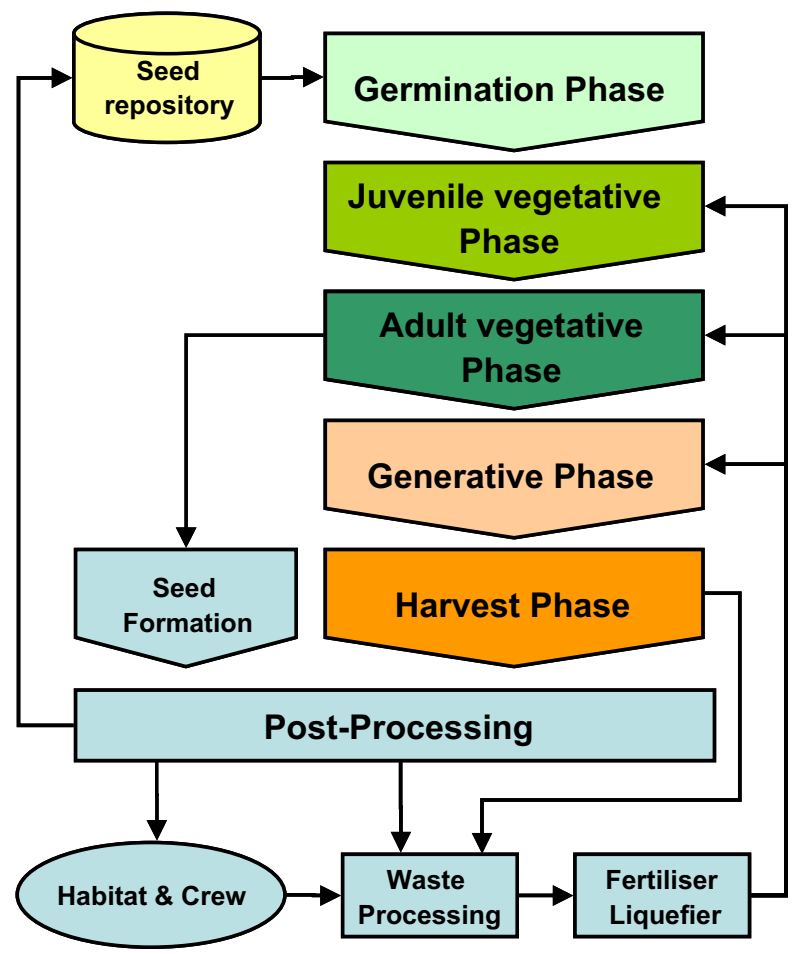

Fig. 5. Life cycle phases of plants (credit: DLR).
(PPF) of $400-450 \mu \mathrm{mol}^{-2} \mathrm{~s}^{-1}$ [35]. On the other hand, wheat needs low rH values ( $\sim 60 \%$ ) for optimal growth but high light levels (PPF) of $750-800 \mu \mathrm{mol}^{-2} \mathrm{~s}^{-1}$ [36].

The process for growing plants in a technical greenhouse is as follows: Out of a seed repository, a defined number of seeds (of one selected plant type) are taken in order to begin the growing cycle. The Germination Phase (G) of the plant usually takes place in a special climate controlled chamber with warm, high humidity conditions. Additional seeding illumination can be provided, as required by individual plant species.

When the seed has developed its first cotyledons (germ layers) as well as its first roots and is ready to begin with the first photosynthesis processes, it is implanted in the primary grow channel. During this Juvenile Vegetative Phase $(\mathrm{J})$ the plant forms its first branches and leaves, and the root system is continuously evolving. The growing conditions required during this phase are high levels of air humidity, initial illumination, and the first addition of low concentrations of nutrient mixtures. Most of the plant growth takes place during the Adult Vegetative Phase (A). Here the plant develops most of its branch and leaf system. The requirements for higher nutrient levels increase while requirements on air humidity levels decrease. During the Generative Phase $(\mathrm{Ge})$ the plant develops one or more fruiting bodies. Requirements towards nutrient levels remain at high levels, while often the composition of the required mixture changes. Once the crops reach a certain level of readiness, the plants enter the Harvest Phase $(\mathrm{H})$. This phase can be short (where all crops are harvested at one time) or an incremental harvest as fruit ripens.

Some plants skip the Generative Phase (Ge) because the edible crop is the leaves (e.g. lettuce). These plants, therefore, need to pass through the seed formation step in order to close the production loop. To accomplish this, some plants are taken out of the nominal production process

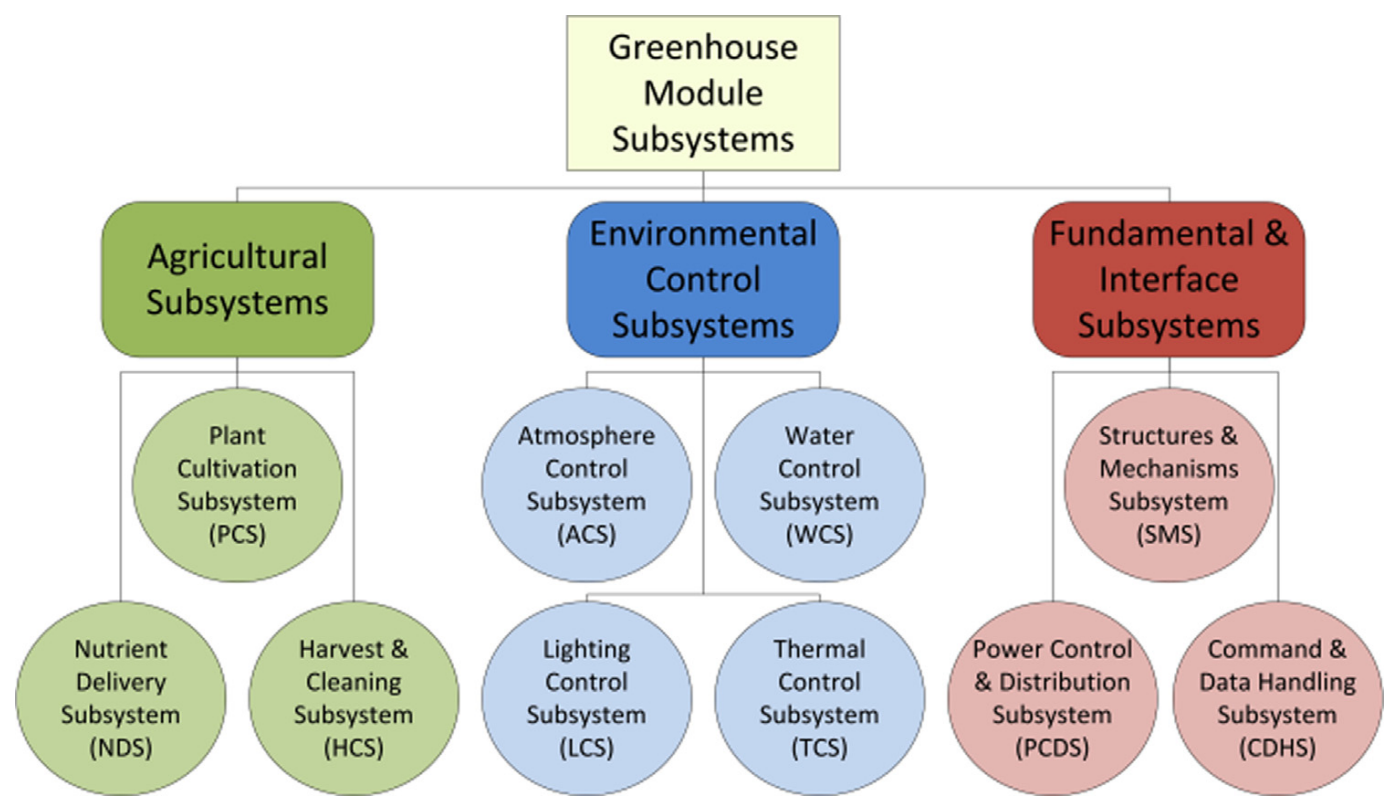

Fig. 6. Possible subsystem overview of necessary subsystems (credit: DLR). 
and are cultivated separately in the seed formation growth unit. Here, the plants can accomplish their biological life cycle in order to produce new seeds that can be used to restock the seed repository. With relatively short mission durations in mind and considering the low mass and volume of some seeds (e.g. carrot has a ThousandSeed-Weight [TSW] of $\sim 11.5 \mathrm{~g}$ ), it seems unnecessary to establish a seed formation growth unit [37]. Nevertheless, for long mission durations and following the premise of closed-loop systems, the generation of new seeds becomes a necessity.

After a successful harvest the crops are relocated to the Food Processing Facility (FPF) where they are refined towards ready to use products. The FPF can be seen as an extended kitchen, where different production steps are executed (e.g. cleaning, cutting, peeling, extracting, disinfecting, sterilizing, boiling, drying, dehydrating, packing and storing). This way, the FPF functions as an interface between the in situ greenhouse and the habitat, including the crew.

During this work phase, some seeds (e.g. wheat) are obtained from the fruit bodies, dried and stored in the seed repository so that they can be used for a new generation of plants. The remaining non-edible biomass is composted together with other bio waste materials of the habitat in order to produce new liquid fertilizer.

System Analysis for Planetary Greenhouses: Comparable to spacecraft, greenhouse modules can be divided into several subsystems. These usually interface with other relevant systems, such as: Air, Food, Thermal, Waste, Water, Crew, In-Situ Resource Utilization, Integrated Control, and Power [31].

The selected approach detailed here (Fig. 6) illustrates a classification of subsystems and their tasks, which has been elaborated within the DLR research group over the last several years. The breakdown structure has proven quite useful in order to cover all necessary systems involved in optimal plant growth.

\subsection{The Agricultural Subsystems}

The first group, the Agricultural Subsystems, encompasses all subsystems directly related to the plants. In that group, the Plant Cultivation Subsystem (PCS) supports the plants during all development stages and contains the growth medium for the plants. The plants themselves can be divided into root and shoot zones. The PCS also has to ensure that the plants have stability in the growth medium and grow as desired. The design of the PCS is directly affected by the selected plant cultivation method and growth medium.

The second branch of the Agricultural Subsystem, the Nutrient Delivery Subsystem (NDS), is responsible for the storage and specialized mixture of each plant's nutrient requirements. The nutrient solution has to be distributed to every plant in the greenhouse module in the desired amount and composition. Nutrient production can be part of the NDS of greenhouse modules, but usually this task is fulfilled by the waste treatment system of the habitat.

The task of the third branch, the Harvest and Cleaning Subsystem (HCS), is the provision of all tools and materials that are necessary for harvesting and cleaning the cultivated plants. Therefore, the HCS has to have a waste storage system to temporarily store the inedible parts of plants, which amount to approximately $50 \%$ of the total produced biomass [38], before they are distributed to the waste treatment system of the habitat. As the crop gathered from plants has to be packed after the harvesting and cleaning procedure, the HCS also has to provide the tools for the packaging. Afterwards the packed crop has to be stored, so the HCS has to provide storage volume as well as a refrigerator and a deep freezer for crops that decay more quickly.

\subsection{The Environmental Control Subsystems}

The second group of subsystems, the Environmental Control Subsystems, maintains all the environmental conditions that are required either by humans or plants. The optimal growth environment is especially necessary for the plants to achieve a high yield. Usually the subsystems of this group are combined in the ECLSS of the spacecraft, but it is suitable to split the functions into different subsystems when analyzing greenhouse modules. Here, the Atmosphere Control Subsystem (ACS) monitors and controls the humidity, the composition (e.g. $\mathrm{O}_{2}, \mathrm{CO}_{2}$, and trace gases) and the pressure of the air. It also has to filter the air and assure circulation through the whole greenhouse module. For gas exchange, the ACS of greenhouse modules is connected to the ECLSS of the habitat.

The Water Control Subsystem (WCS) monitors and regulates the water distribution and water quality. The main task of the WCS is the delivery of the desired amount of water to every plant in the greenhouse module to achieve an optimal growth rate. Water quality is also important for the growth rate of plants. The WCS of greenhouse modules have a connection to the habitat's water management system; therefore, the WCS must be capable of storing a defined amount of water for cases of emergency.

The task of the Lighting Control Subsystem (LCS) is to provide and maintain the illumination of the greenhouse module. Lighting for the crew who work there must also be considered. The plants need specific lighting for an optimal growth rate, which depends on the light spectrum, the light intensity, and the illumination phases. Required lighting conditions differ between plants species, so consequently the LCS has to provide the optimal lighting conditions for each plant species for the maximum yield. Electrical lighting systems require an active cooling system to avoid an overheating of the growth area. Such systems have a connection to the TCS and probably the WCS. When the greenhouse module uses the sun as a light source, the LCS has to regulate the irradiation of the sunlight.

In spacecraft, the Thermal Control Subsystem (TCS) maintains the temperature of all components at every time of the mission within their limits [39]. The TCS of greenhouse modules has to fulfill the same functions, where the critical elements for the TCS are the plants. Different plant species have specific requirements for temperature requiring different temperature zones in the 
greenhouse module. The TCS has to maintain the requirements of each zone. Heat exchangers might be used to utilize the produced heat in other processes. The thermal insulation of the greenhouse module is also part of this subsystem, which has to ensure that the heat loss to the environment and to other parts of the habitat is as low as possible to reduce the energy demand of the TCS.

\subsection{The Fundamental and Interface Subsystems}

The third group of subsystems, the Fundamental and Interface Subsystems, constitute the framework of the greenhouse module. The functions of the Structures and Mechanisms Subsystem (SMS) of greenhouse modules and spacecraft are similar. According to Sarafin, et al. the SMS is the mechanical support of all other subsystems and its structures have to withstand all applied loads during the whole mission as well as serve the function of radiation shielding [40]. Furthermore, the SMS is responsible for all mechanisms used in greenhouse modules.

Unlike the electrical power system (EPS) of spacecraft, the Power Control and Distribution Subsystem (PCDS) of greenhouse modules does not generate electrical energy, it only controls and distributes the electrical energy provided by the electrical power system of the habitat [41]. The PCDS has to supply each of the other subsystems with the voltage they need, to assure the subsystems can work as desired. For cases of emergency, greenhouse modules can contain batteries or other power supplies to keep subsystems operable for a short period. The power demand of greenhouse modules depends on the power consumption of its subsystems; in general the Environmental Control Subsystems have the highest demands, especially lighting (LCS) and thermal control (TCS).

The Command and Data Handling Subsystem (CDHS) of greenhouse modules has to fulfill the same functions as in every spacecraft: receiving, validating, decoding and distributing of commands to other subsystems, and gathering, processing and formatting of data as well as data storage. Security interfaces and computer health monitoring are also functions of the CDHS [42]. In order to maintain optimal growth conditions for plants in greenhouse modules, the CDHS has to interpret the signals of several sensors to send suitable commands to each subsystem. The higher the level of automation of the greenhouse, the lower is the required crew time, but also the higher is the complexity of the CDHS. When the CDHS is a physical part of the greenhouse module, it has to be protected against the high humidity and temperature inside the greenhouse.

Depending on the greenhouse module design approach one can also combine different subsystems, since their functions are related to each other. Water control (WCS) and nutrient delivery (NDS) can be merged into one fluid delivery subsystem, because the nutrients are solute in water and supplied to the plants. The atmosphere and thermal control subsystems (ACS and TCS) also have strong synergies with respect to environmental control. Plant cultivation (PCS) can also be part of the overall structures and mechanisms subsystem (SMS).

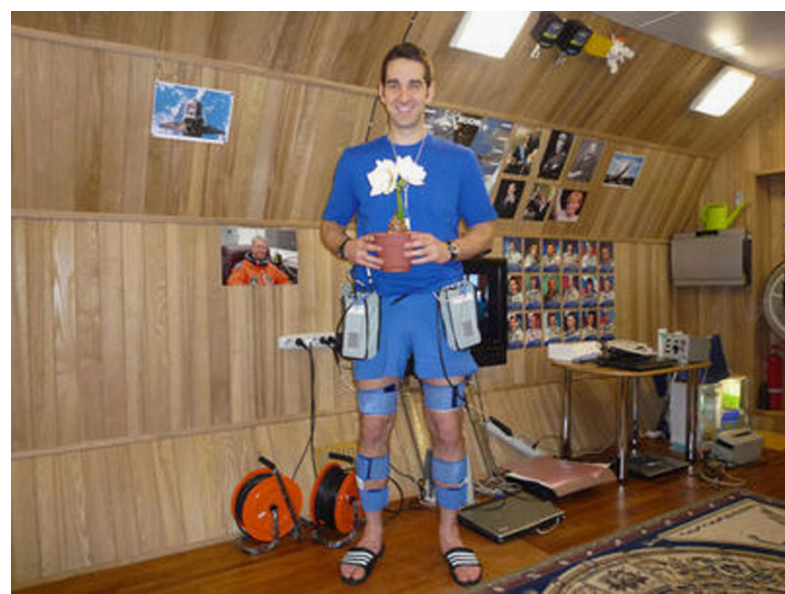

Fig. 7. Oliver Knickel in Mars500 (credit: ESA).

\section{Greenhouses and the human factor}

Even if systems for lighting and watering plants are automatic, there is still a need for certain monitoring and maintaining of those systems. The crew will, for example, monitor for changes in humidity, or the build-up of ethylene waste product, normally removed on Earth by atmospheric and weather conditions ([23] p. 184). Time allocated for "functions related to food," such as food production, preparation, and waste disposal, may require a significant portion of crew time (31 p. [20]).

Humans also have responsibility for plant reproduction, and studying the environmental effects on different plants over generations helps to improve viability of seeds. Not until 2001, after close to 30 years of experimentation and refinements to space hardware to improve trace gas monitoring, did astronauts achieve a significant milestone, a $70 \%$ viability rate ([23] pp. 184-185). Early missions had for the most part yielded only seeds and germs of wheat that were sterile ([23] pp. 182-183). In the case of the Svet greenhouse experiments in 1997, the seeds of space-born plants diminished in strength and viability in each generation ([23] p. 184). Space-born seeds can be genetically mutated, like the tomato seeds brought to the ISS on STS111, which when returned to Earth after 6 years germinated faster in their terrestrial soils, a benefit ([23] p. 185).

In addition to technical requirements for maintenance of plants, for pruning they need human stewards. Cosmonaut Vladimir Kovalenok tended his onions by keeping rot at bay, trimming stalks that were unhealthy, which promoted growth in the plant. He called his method the "simple peasant's way." [11]. When dealing with more complex plants in the future such as fruit bearing plants, it is clear that human care of plants will be become even more important; however, crew time must be simplified in this area as much as possible to minimize requirements.

In space the "success" rates of plants will be determined not simply by whether they live or die, but whether they have the possibility to thrive. Astronauts will likely use plants' growth and health to measure their own, as was seen to happen in the Mars500 experiment in 2009 when Oliver Knickel created his own palliative for passing 
the long time in cramped quarters, with a kind of "letter home" in the form of a flower (Fig. 7). From Knickel's diary: "Spring has already reached the inside of our module since the [bulbs] we took inside our greenhouse a month ago ... sprang up and are already nice and big... I promised my girlfriend to plant a flower for her, [so] I was very pleased to see that the one I dedicated to her has turned out to become a real beauty." [43].

It is already determined that twenty-four hour schedules result in better crew performance and health due to maintenance of circadian rhythms ([44] p. 137). In space, away from normal diurnal cycles and in the absence of seasons, plants will provide an important measure for human life. Plants have the ability to mark time, though it is "slow time."

The emptiness of space can be psychologically difficult as detailed in Kanas, Space Psychology and Psychiatry. A type of anxious depression can manifest-“Long Eye Syndrome"-where someone gazes out of the window back toward Earth for long periods of time [44]. One can only guess that on a mission to Mars such a condition would become more extreme. References to time, the seasons, and the life cycle itself, will all but disappear.

The need to mark time has already been noted on ISS missions. Vladimir Gushin, a psychologist at Russia's Institute for Biomedical Problems stated in an interview, "Confinement on the space station isn't the problem, it's a lack of stimuli... Plants are one of the opportunities that makes [astronauts] feel something is changing, that nature is with them, a piece of earth is with them... That gives them the feeling ... of Earth, of life. From this [perspective], nothing can substitute for plants." [16].

Highly technical spaces are esthetically neutral, supremely functional, "cold" (devoid of personalization), and often visually crowded. Plants add natural colors without over-saturating such spaces. "A limited variety of color of medium brightness and saturation" are recommended by NASA, according to Kanas, including "cinnamon, beige, cream, maize, straw, ivory, pale yellow, and blue." ([22] p. 135). The Soviet stations Salyut and Mir space station integrated a muted color system to mimic Earth orientation (walls and ceiling), but also to improve mood.

More so than to their color/s the psychological benefits of plants can be attributed to their taste and smell. Dr. Fred Davis, AgriLife Research Faculty Fellow at the Texas A\&M University, researches low-pressure controlled food production environments for NASA. He commented that for astronauts "just biting into something with some turgor [i.e. skin membrane] to it, and not having a diet limited to reconstituted foods has important psychological benefits." [45]. NASA is still conducting rigorous testing on space-grown plants before approving they be eaten by astronauts [34].

Food systems in space have changed to a large degree over the past years. While for the early astronauts "food was just taking medicine" now astronauts are experimenting with "space-cooking" in order to increase the variety ([17] p. 213, 218). Vickie Kloeris, manager of ISS food systems at the NASA Johnson Space Center in Houston says, "Food is a comfort that [the astronauts] would like to feel they have some input on or some control over... [It's]
psychological-I don't know if we've flown anyone to the station who hasn't been concerned about their food." [46].

In contrast to the current strategy of not bringing strong smells into space in order not to disturb other crewmembers, the introduction of recognizable scent and tastes (through food) is being considered ([46,15] p. 185, 195). The integration of herbs, vegetables and fruits like tomatoes, spinach and strawberries provide extra nutrients that are absorbed differently than supplemental vitamins, provide natural fiber, and create the possibility for astronauts to personalize their menus. The emphasis in future endeavors will be on high-caloric foods that require little or no processing [34].

Documented testimonials about how noxious space capsules can smell suggest plants have another important function for humans in space. Plants that suggest "freshness" can normalize the environment of astronauts on long-term missions and deal with a certain amount of indoor "air pollution" cased by humans themselves. We must remember the early days of space travel through the words of Apollo 15's Jim Irwin, who attested, "I began to smell like a restroom... It got so I couldn't stand my own company." ([47] p. 99).

Davis reports that a greenhouse at a U.S. Antarctic base which supplies salad bowl crops is "one of the most popular places on the base, where crew members will retreat from the cold, white, barren, snow-capped landscape to recharge, rest and nap in hammocks stretched across the green visual of live, growing, green plants." The mental image is almost Edenic with all "those bright, light colors, aroma, texture and flavor of plants." Davis concludes, "Small wonder that the greatest pastime in the U.S. is gardening. It will also be an important activity as humankind colonizes space during the 21st Century." [45].

\section{Architectural integration of greenhouses into the habitat system}

To recap, the unique physical requirements put on greenhouse design for space architecture have to allow optimum production of food, fresh water and oxygen, include vital recycling processes to minimize waste production, and at the same time provide maximum mental health benefits for the crew with minimum mission costs. New greenhouse technologies thus need to combine agricultural and gardening requirements. One way to do this is to revise the growing chambers for space habitats on both a design-level and in terms of philosophical approach.

\subsection{Personal use of greenhouses}

While the incorporation of time saving technical systems will be important for the smooth operation of greenhouses, personal use of greenhouses and recreational plant caretaking should be anticipated in design, allowing for the preferences of individual crewmembers. This time should not be essential to the physical health of the crew but rather endeavored within small windows of time primarily for plants' overall wellbeing. 
There is a reported benefit to astronauts to have time away from the heavily structured regimen of the spacefarer's workload ([48] p. 137). The effect of growing plants is measurable in that the difference in brain activity is significant between the high frequency beta waves of rational thought necessary for day-to-day work operations and the slower alpha and theta waves produced by repetitive activities conducted in a relaxing way, such as planting seeds, watering, and tending to plants ([49] p. 80).

Astronauts confirm the importance of tending plants to keep themselves occupied during their free time and to encourage interaction of the crew with living beings in a technologically mediated habitat [17]. On Salyut 1, the first flax seed sprouts were tended to devotedly by crew members Viktor Patsayev and Vladislav Volkov, who found a common source of emotional connection through the plants. "These are our pets," were Patsayev's words. "They are our love," said Volkov [11]. Because plants are deeply rooted as references within all human cultures, it seems that greenhouses can form an interface with other leisure forms like music, art and literature, and plants can serve as points of connection between crewmembers, especially on international missions.

On typical short flights astronauts are too busy to spend much time on contemplative activities, as attested to by Gemini 10 astronaut Michael Collins; notes Oliver, “After laboring across 20 feet of open space to retrieve an experiment package from an inert Agena rocket, Gemini 10 astronaut Michael Collins imagined what he might have said about the experience: 'I found God outside my spacecraft. Wrong, I didn't even have time for look for Him."” ([47] p. 98). However, on long-term space missions or on Moon or Mars bases, there will be more down time than has been experienced so far by astronauts, and one of the challenges will be boredom and monotony ([44] p. 137).

Established in two important works by the French philosopher of science Gaston Bachelard, "The Experience of Space in Contemporary Physics" and The Poetics of Space, contemplation is a key activity for the relaxation of the mind. The "seeds" born in moments of deep-looking (creating alpha brain waves) or daydreaming (creating theta brain waves) give rise to instantaneous images, which Bachelard defines as poetic because they simply appear. These images have no historical antecedent of thought, emerging suddenly and as whole images. Bachelard contrasts poetic thought with scientific rationalism, which necessitates building upon past knowledge using logic in order to think through the next steps in a developing chain of discoveries. Bachelard writes that there can be an "ecstasy" found in the newness of poetic thought, "a sudden salience on the surface of the psyche." ([50] p. xv). The inherent nature of the poetic is its surprise.

For the astronaut, the surprise of poetic thought-like the moment of a seed's first visible germination-would be a key counterbalance to the routines of space life, to boredom and the doldrums, an antidote to the quiet internal suffering of being a human contained in such a small space. Through poetry, there is expansion of thought, as in the works of astronaut Dr. Story Musgrave: “... Sculptured by the seasons, listening to Nature's reason, Grew I, rooted in the ether." [51].

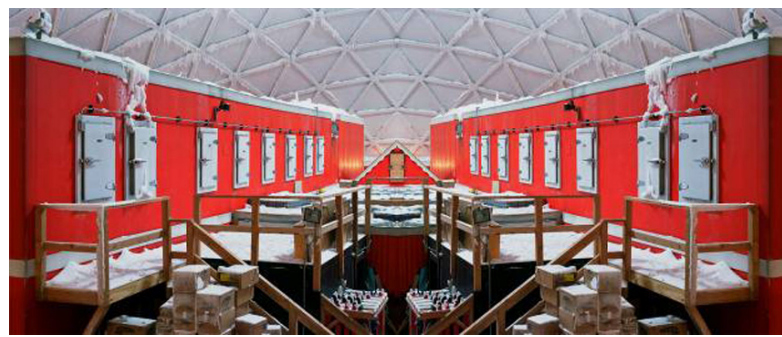

Fig. 8. Antarctic greenhouse entrance in the Dome (now dismantled), Amundsen-Scott Research Station (credit: V.A.L.I.S. series 2005-7 (C) Connie Samaras).

Kanas and Manzey report that territorial behavior can result when people are living long-term in confined spaces and under "prolonged isolation," if private spaces are not adequate ([22] p. 134). It can be seen how astronauts having smaller, personal break-away greenhouse systems, like his or her own little "plot" of land, could differentiate personal spaces from communal ones and thus prove to promote crew cohesion.

As reported by the Horticultural Society of New York, which since 1989 has used plant-cultivation therapy with inmates on Rikers Island, "stress reduction, mood improvement, alleviation of depression, social growth, physical and mental rehabilitation" are all possible using plants in enclosed spaces with limited movement and choice available [34]. As on Earth, it will be important for people living in enclosed spaces off-planet to feel there is some choice of location for activities such as eating, work, rest/relaxation and exercise (Fig. 8).

\subsection{Integration of a new subsystem: the mobile plant cultivation subsystem}

In the paper "Greenhouse design integration benefits for extended spaceflight," Haeuplik-Meusburger, Peldszus and Holzgethan have touched upon the topic of Japanese design philosophies and listed several design integration concepts, as follows: (a) Surrogate views through "green" windows, (b) Spatial enhancement: stretching the view using illusion and perspective, and (c) Individual interaction through modular growth units [6].

An adequate living representation of nature (flora and possibly fauna) in isolated environments such as space habitats could, most immediately, be of importance on long-term missions to Mars, where the visual connection to Earth is broken and the greenhouse is the only "living" area in an otherwise technical surrounding. (Fig. 9a and b) Further in extreme environments, where the inclusion of windows means a potential risk for human beings in the habitat, "surrogate windows" (a), such as an integrated greenhouse could add to habitability.

Haeuplik-Meusburger, Peldszus and Holzgethan discuss the Japanese methodology of "Stretching the view" (b) with findings by space psychiatrist Kanas [6,17], who reports that additions of viewing layers can create sightlines that can enlarge the perceived space of space capsules, which psychologically "compensate for the 


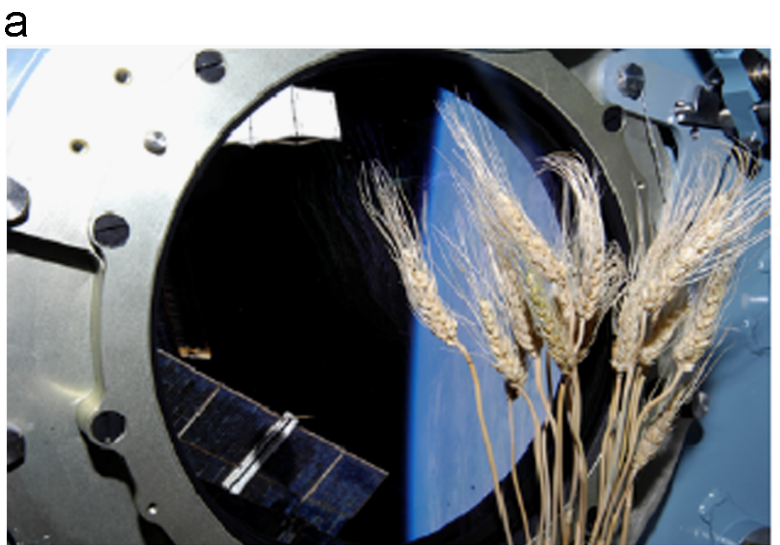

b

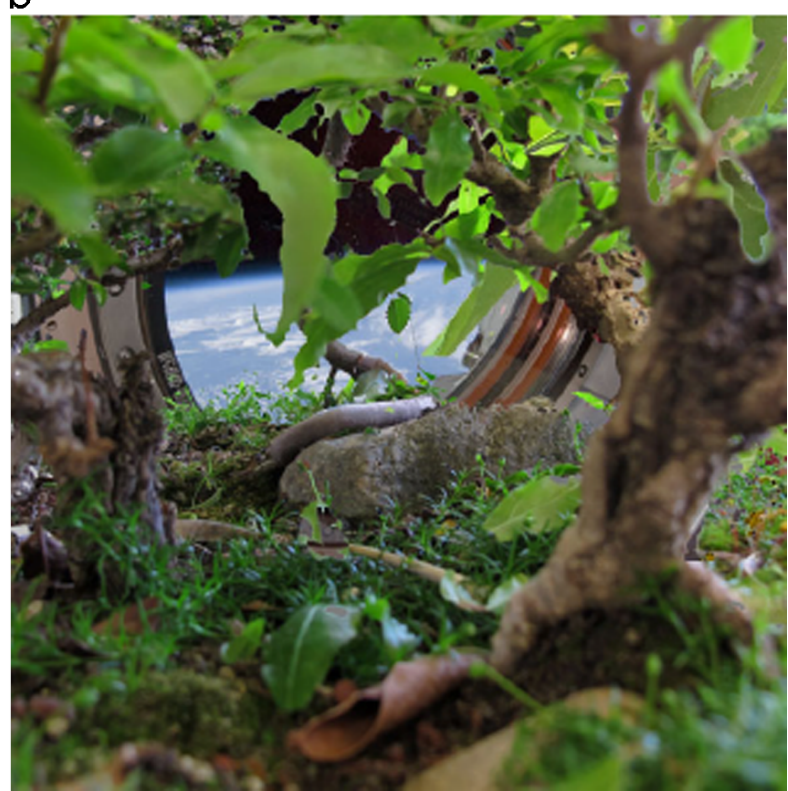

Fig. 9. (A) Artistic picture of the wheat harvest from the Lada module during Expedition 22 (image by crew member Maxim Suraev; NASA). (B) Visualization of a spatial enhancement using a Mobile Plant Cultivation Subsystem (C C. Paterson).

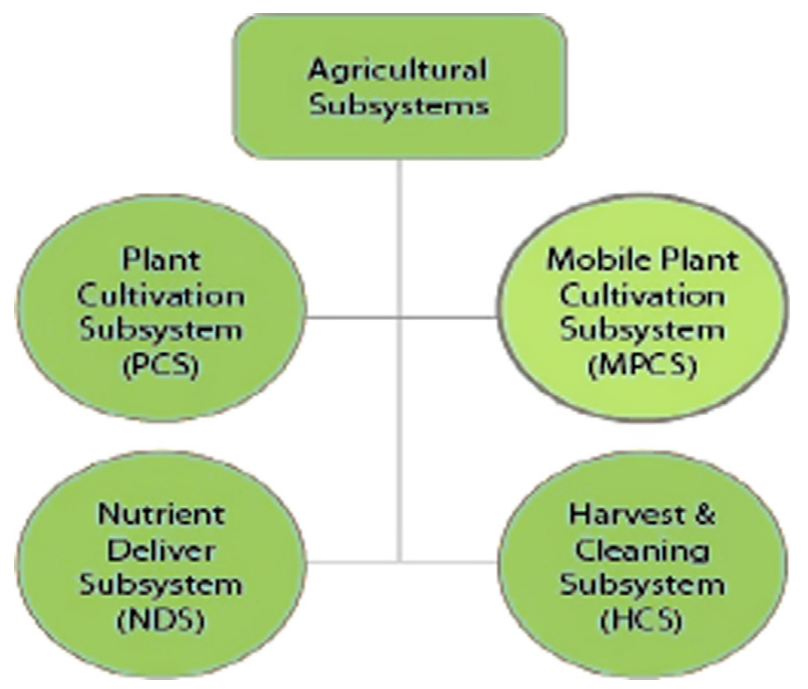

Fig. 10. Mobile Plant Cultivation Subsystem (diagram: S. HäuplikMeusburger, based on DLR diagram). effects of the otherwise decreased range of environmental cues in a space habitat." ([44] p. 135).

An addition to the classification of greenhouse subsystems as proposed in Fig. 6 would include individual mobile growth units (c). These "dock" to a larger greenhouse facility and would further enhance living conditions by providing food supplements or esthetic improvements to living spaces. These individual mobile growth units would be easily incorporated into the Agricultural Subsystems of modular greenhouse design requirements as the Mobile Plant Cultivation Subsystem (MPCS) (Fig. 10).

While the Plant Cultivation Subsystem (PCS) supports plants during all development stages and contains the growth medium for the plants in the main chamber, its mobile version (MPCS) supports the same activities when separated from the main greenhouse chamber, for example, during travel onto extraterrestrial surfaces, in common spaces, or private habitation spaces.

A planetary greenhouse system, for example, can host these mobile greenhouses (MPCS) together and, when connected, the MPCS is part of the automated system. This option is important because of human-related aspects. In case the crew is on a rover mission, is sick, busy with a pressing or emergency matter, or in between regular maintenance schedules, the MPCS is no extra work. The time that the mobile plant unit would not be connected, and thus not sufficiently supplied, would have to be limited to and dependent upon the requirements of particular plants.

The development of the MPCS will require modular architectural solutions, which can be informed by experiments being conducted over the next few years growing plants in space.

\subsection{A look towards the future of Space Gardens}

NASA Ames planetary scientist Chris McKay, a longtime advocate for planetary exploration, is supporting the Lunar Oasis venture, where a private corporation Odyssey Moon Ltd. intends to grow the first plant on the Moon. McKay says, "The first plant to grow from seed and complete its life cycle on another world will be a significant step in the expansion of life beyond the Earth." This "Moon flower" is considered by the company to have "symbolic as well as scientific importance." [48].

McKay points to the idea of a simple flower on the Moon as a benchmark. Much like having the first animals and then humans orbit the Earth, the first plant grown on an extra-terrestrial surface heralds a paradigm shift-the dawn of a new Garden [50,5].

Douglas Trumbull's 1972 film Silent Running is a visualization of the latter, though with an apocalyptic ending uncharacteristic of American directors. Unwittingly, the film has provided space architecture and space greenhouses with a futuristic vision for space greenhouse architecture in its Buckminster Fuller geodesic domes. These extremely strong, flexible and resilient structures have continuous tension across a network of simple, rigid triangles and repeat fundamental patterns in molecular carbon-based structures (Fullerene). In his 2010 TED talk "Calculus in Architecture," international renowned 
architect Greg Lynn started his lecture referring to nature as an example of "good architecture." Architects, designers and engineers have studied principles in nature in order to find innovative solutions over centuries. The research fields of bionics and biomimetics, wherein systems and design solutions from nature are studied in order to develop innovative technical and engineering solutions would be expanded by studying plants grown in different gravity than Earth. "Scientifically it will be very interesting to understand the effects of the Moon and one sixth gravity on plant growth," said Dr. Volker Kern, who conducted plant growth experiments on Space Shuttle missions [47].

\section{Conclusion}

Habitability issues in man-made extra-terrestrial environments do not just demand technical or architectural responses, but also consideration of the unique philosophical, emotional, existential and esthetic conundrums of living and being human in the most extreme circumstances of limitation. Having hope in the future of a plant, no matter how small, requires a positive outlook; the health of the plant requires cooperation. Both of these qualities are important requirements for the cohesion and wellbeing of crew members. Plants can "humanize" technical spaces because they are not functions of human will but symbiotic partners. Any design program for space greenhouses needs to integrate plants within the crew's daily living and working environments, which can be done with the modest expansion of technical greenhouse systems to incorporate detachable, personal modular units.

\section{References}

[1] Rebecca Reider, Dreaming the Biosphere: The Theater of All Possibilities, University of New Mexico Press, Albuquerque, NM, 2009.

[2] Silverstone, Development and Research Program for a soil-based bioregenerative Agriculture System to feed a four person crew at a Mars Base.

[3] O. Monje, G.W. Stutte, G.D. Goins, D.M. Porterfield, G.E. Bingham, Farming in space: environmental and biophysical concerns, Adv. Space Res. 31 (1) (2003) 151-167.

[4] C. George, Marshall Space Flight Center, Skylab, Classroom in Space, in: B. Lee (ed.), National Aeronautics and Space Administration, Summerlin. NASA SP-401, Washington, D.C., 1977.

[5] D.M. Porterfield, et al., Spaceflight hardware for conducting plant growth experiments in space: the early years 1960-2000, Adv. Space Res. 31 (1) (2003) 183-193.

[6] Sandra Häuplik-Meusburger, Regina Peldszus, Verena Holzgethan, Greenhouse design integration benefits for extended spaceflight, Acta Astronaut. 68 (1-2) (2011) 85-90.

[7] B.J. Bluth, M. Helppie, Soviet Space Stations as Analogs, NASA Headquarters Washington, NASA Grant NAGW-659, 1987.

[8] M.A. Perino, C. Lobascio, S. Pastro, Greenhouse: A Strategic Element to Support Humans in Space, in: M. Rycroft (Ed.), Beyond the International Space Station, Kluwer Academic Publishers, Dordrecht, 2002, pp. 119-126.

[9] Mary E. Musgrave, Anxiu Kuang, Sharon W. Matthews, Plant Reproduction During Spaceflight: Importance of the Gaseous Environment, Planta, vol. 203, Springer, Berlin Heidelberg, 1997, 177-184.

[10] Gordon Hooper, Missions to Salyut 4, Spaceflight 42 (1977) 64.

[11] Robert Zimmerman, Growing pains: it's the one area of space science in which you get to eat the experiment, Air \& Space Magazine, September 01, 2003.

[12] Robert Zimmerman, Leaving Earth: Space Stations, Rival Superpowers, and the Quest for Interplanetary Travel, Joseph Henry Press, Washington, D.C., US978-0309085489, 2003.
[13] Valentin Lebedev, Diary of a Cosmonaut - 211 days in Space. s.l., Bantam Air \& Space Series, New York, 1982.

[14] NASA: Growing Vegetables in Space 〈http://www.nasa.gov/mission_ pages/station/research/10-074.html .

[15] Doug Messier, Orbitec's Veggie Designed to Give ISS Astronauts Fresh Salads, 2-May 2012 [Online].

[16] Sheri Quinn, Plants as important in space as on Earth, Voice Am. (2008), 〈http://www.voanews.com/content/a-13-2008-04-08-voa3266747192/562482.html>.

[17] Sandra Häuplik-Meusburger, Architecture for Astronauts, Vienna: Springer Wien, New York, 2011.

[18] Orbitec, Astro Garden and Space Garden, 2012 〈www.orbitec.com〉 [Online].

[19] 〈http://modernfarmer.com/2013/09/starship-salad-bar/〉.

[20] ESA, ESA's Greenhouse in Space, Space for Life - Human Spaceflight Science Newsletter, July 2011.

[21] Wubbo, Ockels, Interview not published [interv.] Sandra HaeuplikMeusburger, Delft, The Netherlands, March 2012.

[22] Colin Foale, Waystation to the Stars - The Story of Mir, Michael and Me, Headline Book Publishing, London, GB0 74726331 0, 1999.

[23] Javier Casado, Horticulture in space, Spaceflight - The Magazine of Astronautics and Outer Space, Vol 48(5), May 2006.

[24] Yamashita et al., An overview of challenges in modeling heat and mass transfer for living on Mars, 2006.

[25] M. Nelson, W.F. Dempster, J.P. Allen, Integration of lessons from recent research for "Earth to Mars" life support systems, Adv. Space Res. 41 (5) (2008) 675-683. http://dx.doi.org/10.1016/j. asr.2007.02.075, (Published by Elsevier Ltd. on behalf of COSPAR).

[26] Federico Maggi, Céline Pallud, Space agriculture in micro-and hypogravity: a comparative study of soil hydraulics and biogeochemistry in a cropping uniton Earth, Mars, the Moon and the spacestation, 2010, http://dx.doi.org/10.1016/j.pss.2010.09.025 (Elsevier Ltd. All rights reserved).

[27] H. Liu, C.Y. Yu, N.S. Manukovsky, V.S. Kovalev, Yu L. Gurevich, J. Wang, A conceptual configuration of the lunar base bioregenerative life support system including soil-like substrate for growing plants, Adv. Space Res. 42 (6) (2008) 999-1166.

[28] Masamichi Yamashita, Hirofumi Hashimoto, and Hidenori Wada, On-Site Resources Availability for Space Agriculture on Mars, 2009, pp.517-542.

[29] Federico Maggi, Céline Pallud, Martian base agriculture: the effect of low gravity on water flow, Nutrient Cycles and Microbial Biomass Dynamics, 2010.

[30] Federico Maggi, Céline Pallud, Space agriculture in micro- and hypogravity: a comparative study of soil hydraulics and biogeochemistry in a cropping unit on Earth, Mars, the Moon and the space station, 2010.

[31] A.J. Hanford, Advanced life support baseline values and assumptions document, Document no. CTSD-ADV-484A, Crew and Thermal Systems Divison, NASA-Lyndon B, Johnson Space Center, Houston Texas, JSC 47804, 16 August 2004.

[32] D. Bubenheim, M.E. Bates, M.T. Flynn, An approach for development of regenerative life support systems for human habitats in space, NASA SAE Technical Paper 951730, 1995.

[33] NASA, "Cosmic Cuisine", National Aeronautics and Space Administration, FS-2005-10-055-JSC, Houston, Texas, 2005.

[34] 〈http://modernfarmer.com/2013/09/starship-salad-bar/〉.

[35] S. Sasidharan, MELiSSA reference crop data, Issue 1 Rev 1, ESA 2013, 19/04/2013.

[36] R.M. Wheeler et al., Crop production for advanced life support systems - observations from the Kennedy Space Center Breadboard Project, NASA-KSC; TM-2003-211184, February 2003.

[37] J.L. Mangal, B.R. Barta, G.R. Singh, Effect of irrigation intensities and nitrogen and phosphorus interaction on seed production of radish (Raphanus sativus L.), Haryana J. Hortic. Sci. 17 (1988) 97-101.

[38] D.L. Bubenheim, T. Wydeven, Approaches to resource recovery in controlled ecological life support systems, Adv. Space Res. 14 (11) (1994) 113-123.

[39] David G. Gilmore, Brian E. Hardt, Robert C. Prager, Eric W. Grob, Wes Ousley, "Thermal," in Space Mission Analysis and Design, Microcosm Press \& Kluwer Academic Publishers, Torrance, California, 2005 (Chapter 11.5).

[40] Thomas P. Sarafin, Peter G. Doukas, James R. McCandless, William R. Britton, "Structures and Mechanisms," in Space Mission Analysis and Design, Microcosm Press \& Kluwer Academic Publishers, Torrance, California, 2005 (Chapter 11.6).

[41] Joseph K. McDermott, "Power," in Space Mission Analysis and Design, Microcosm Press \& Kluwer Academic Publishers, Torrance, California, 2005 (Chapter 11.4). 
[42] Richard T. Berget, "Command and Data Handling," in Space Mission Analysis and Design, Microcosm Press \& Kluwer Academic Publishers, Torrance, California, 2005 (Chapter 11.3).

43] Mars500 Diary: Testing, Testing Status Report Source: European Space Agency, Posted Tuesday, May 5, 2009 〈http://spaceref.com/ news/viewsr.html?pid=31178 $>$.

[44] N. Kanas, D. Manzey, Space Psychology and Psychiatry, Springer, The Netherlands, 2003.

[45] David Morgan, Growing food for space travelers, Urban Dirt, August 2004.

[46] Jim Romanoff, When it comes to living in space, it's a matter of taste, Scientific American, March 10, 2009.

[47] Kendrick Oliver, To Touch the Face of God: the Sacred, the Profane and the American Space Program, 1957-75, Johns Hopkins University Press, Baltimore, Maryland, 2013.
[48] Paragon Space Development Corporation, Media Release: Google Lunar X Prize Contender Odyssey Moon Partners with Paragon Space Development Corporation to Grow first Plant on the Moon, Odyssey Moon, Tucson, Arizona, March 27, 2009.

[49] Leandre Poisson, Gretchen Vogel Poisson, Solar Gardening: Growing Vegetables Year-Round the American Intensive Way, Chelsea Green Publishing, Vermont, US, 1994.

[50] Gaston Bachelard, The Poetics of Space, Beacon Press, Boston, US978-0807064733, 1994.

[51] Story Musgrave, Poem: Story's Prelude, s.l., June 1988 〈http://www. spacestory.com/spacial.htm $\rangle$. 